\title{
Qualitätsindikatoren für die Kolonoskopie
}

Für die Verhinderung von sog. Intervallkarzinomen bei der Screeningkolonoskopie ist der Anteil entdeckter Adenome der entscheidende prädiktive Parameter, nicht dagegen das Erreichen des Zökums.

— Ziel einer Screeningkolonoskopie ist es, kolorektale Adenome frühzeitig zu erkennen und zu entfernen, ehe sie sich zu Karzinomen entwickeln. Intervallkarzinome, d.h. kolorektale Karzinome, die im Zeitraum zwischen der Screeningkolonoskopie und der entsprechend den Leitlinien terminierten Überwachungskolonoskopie auftreten, müssen daher als Scheitern der Screening-Intension angesehen werden. Die Ursachen für dieses Scheitern können vielfältig sein und reichen von einer methodisch immanenten falsch negativ Rate (z. B. Lokalisation des Adenoms hinter einer Falte) über eine ungenügende Darmreinigung bis zu mangelnder Übung oder Sorgfalt des Untersuchers.

Unter den Qualitätskriterien der Fachgesellschaften für die Screeningkolonoskopie rangieren die Adenomdetektionsrate und das Erreichen des Zökums ganz oben. Allerdings wurden diese Parameter nie validiert und es ist nicht bekannt, ob eine Verbesserung bei den Qualitätsindikatoren tatsächlich zu einer höheren Effizienz des Screenings führt.

Jetzt wurden die Daten von 186 Endoskopikern analysiert, die zwischen 2000 und 200445026 Personen im Alter zwischen 40 und 66 Jahren mit durchschnittlichem Risiko für ein kolorektales Karzinom untersucht haben. Untersuchungsdaten von Endoskopikern, die im Untersuchungszeitraum weniger als 30 Kolonoskopien durchführten, wurden von der Analyse ausgeschlossen.

Anhand eines nationalen Krebsregisters identifizierte man die Personen, bei denen in den fünf Jahren nach der Screeningkolonoskopie ein kolorektales Karzinom diagnostiziert worden war. Im Lauf eines Zeitraums von 188788 Personenjahren traten 42 Intervallkarzinome auf. Anhand eines multivariaten Regressionsmodells zeigte sich, dass eine signifikante Assoziation zwischen der Adenomdetektionsrate des Endoskopikers und dem Auftreten eines Intervallkarzinoms bestand.

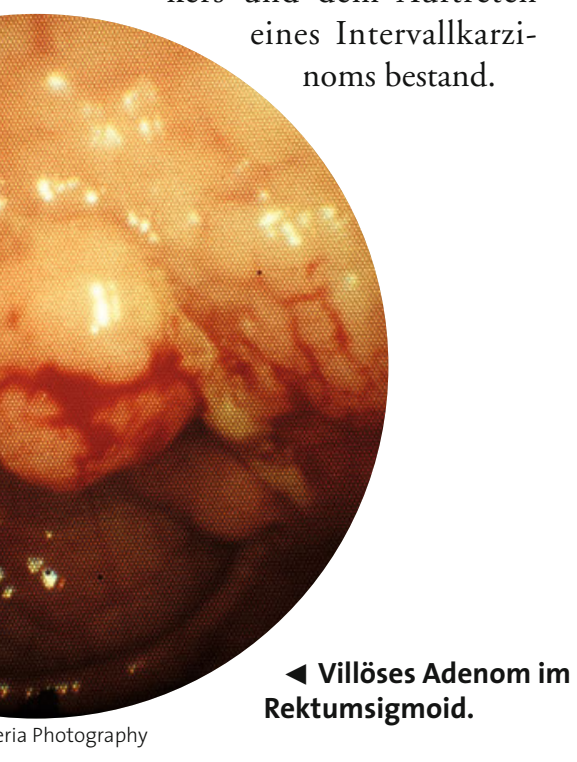

Insgesamt betrug die durchschnittliche Adenomdetektionsrate 12,2\%. Unter den Patienten von Endoskopikern mit einer Adenodetektionsrate unter elf Prozent traten im Lauf von 100000 Personenjahre des Follow up 33,6 Intervall-Karzinome auf. Bei Endoskopikern mit einer Detektionsrate von $\geq 20 \%$ waren es dagegen nur 2,4 Intervallkarzinome pro 100000 .

Dies bedeutet, dass im Vergleich zu den Endoskopikern mit einer Detektionsrate über $20 \%$ in den Kategorien unterhalb dieses Werts ein kumulativer Hazard-Quotient zwischen 10,94 und 12,50 bestand. Interessanterweise spielte die vielfach als Qualitätsparameter angesehene Einsicht des Zökums für das Auftreten von Intervallkarzinomen keine signifikante Rolle.
- M. F. Kaminski et al.

(Korr.: Jaroslaw Regula, MD, Department of Gastroenterology, Institute of Oncology, Roentgen St. 5, Warsaw, Poland, e-mail: jregula@coi.waw.pl): Quality indicators for colonoscopy and the risk of interval cancer. New Engl. J. Med. 362 (2010) 19, 1795-1803

\section{Kommentar}

Die Ergebnisse der Untersuchung unterstreichen die Wichtigkeit einer sorgfältigen und minutiösen Inspektion der kolorektalen Mukosa gerade bei der Screeningkolonoskopie. Andere Faktoren, insbesondere die ineffektive Polypektomie spielten in dieser Untersuchung vergleichsweise eine zu vernachlässigende Rolle. Zwei methodische Details schränken die Aussage der Studie etwas ein. Zum Einen wurden Daten des nationalen polnischen Krebsregisters herangezogen, das einen Erfassungsgrad von $89 \%$ aufweist. Allerdings könnte gerade diese Untererfassung das Ergebnis möglicherweise noch ungünstiger für die Endoskopiker gestalten. Zum anderen wurde die Rückzugszeit des Endoskops in der Studie nicht reduziert, ein Faktor, der in anderen Untersuchungen sich als wichtiger Qualitätsparameter bei der Kolonoskopie erwiesen hat. Bei allen Einschränkungen sollte jeder Screening-Endoskopiker stutzig werden, wenn er bei weniger als $15 \%$ seiner untersuchten Patienten Adenome feststellt. Umgekehrt könnten Patienten ihrem Endoskopiker die Frage stellen, wie hoch seine Detektionsrate denn ist. Auf den einschlägigen Websites von gastroenterologischen Fachpraxen sucht man diese Angabe in der Regel vergebens.

H. S. FüEßL $=$ 